\title{
Graph bisection revisited
}

\author{
Renata Sotirov ${ }^{1}$
}

Published online: 5 July 2017

(C) The Author(s) 2017. This article is an open access publication

\begin{abstract}
The graph bisection problem is the problem of partitioning the vertex set of a graph into two sets of given sizes such that the sum of weights of edges joining these two sets is optimized. We present a semidefinite programming relaxation for the graph bisection problem with a matrix variable of order $n$-the number of vertices of the graph-that is equivalent to the currently strongest semidefinite programming relaxation obtained by using vector lifting. The reduction in the size of the matrix variable enables us to impose additional valid inequalities to the relaxation in order to further strengthen it. The numerical results confirm that our simplified and strengthened semidefinite relaxation provides the currently strongest bound for the graph bisection problem in reasonable time.
\end{abstract}

Keywords Graph bisection · Graph partition · Semidefinite programming · Boolean quadric polytope

\section{Introduction}

The graph bisection problem (GBP) is the problem of dividing the vertices of a graph into two sets of specified sizes such that the total weight of edges joining different sets is optimized. The GBP is an NP-hard combinatorial optimization problem, see Garey et al. (1976). It has many applications such as VLSI design (Lengauer 1990), parallel computing (Biswas et al. 2000; Hendrickson and Kolda 2000; Simon 1991), network partitioning (Fiduccia and Mattheyses 1982; Sanchis 1989), and floor planing (Dai and Kuh 1987). Graph partitioning also plays a role in machine learning (see e.g., Li et al. 2015) and data analysis (see e.g., Pirim et al. 2012).

There are several SDP relaxations for the GBP with matrix variables of different orders. In particular, there are relaxations whose matrices have orders $n, 2 n$, and $2 n+1$, where $n$ is

Renata Sotirov

r.sotirov@uvt.nl

1 Tilburg University, Warandelaan 2, 5000 LE Tilburg, The Netherlands 
the order of the graph. An SDP relaxation with a matrix variable of order $n$ is introduced by Karisch et al. (2000). The same relaxation is used by Feige and Langberg (2001), and Han et al. (2002) to derive approximation algorithms for the GBP. Another SDP relaxation with a matrix variable of order $n$ that is derived from an SDP relaxation for the more general graph partition problem is introduced in Sotirov (2014). In Sotirov (2014) it is also proven that the above mentioned SDP relaxations of order $n$ are equivalent.

Wolkowicz and Zhao (1999) derived an SDP relaxation with a matrix variable of order $2 n+1$. This SDP relaxation with additional nonnegativity constraints dominates the SDP relaxations with matrix variables of order $n$, see De Klerk et al. (2012); Sotirov (2014).

The GBP can be seen as a special case of the quadratic assignment problem (QAP). De Klerk et al. (2012) exploited this to derive an SDP relaxation for the GBP from an SDP relaxation for the QAP, which however reduces to a much smaller semidefinite program than the original QAP relaxation (see also De Klerk et al. 2012). In particular that relaxation contains matrix variables of orders $n$ and $2 n$. In Sotirov (2014), it is proven that the QAPbased SDP relaxation for the GBP is equivalent to the strongest SDP relaxation, that is the SDP relaxation with nonnegativity constraints from Wolkowicz and Zhao (1999).

For specific families of (symmetric) graphs, De Klerk et al. (2012) improved the QAPbased SDP relaxation for the GBP by adding a constraint that fixes one vertex of the graph. Finally, in van Dam and Sotirov (2015) the SDP relaxation for the GBP from De Klerk et al. (2012) was further strengthened by adding two constraints that correspond to assigning two vertices of the graph to different parts of the partition. Both fixing-based strengthening perform well on highly symmetric graphs.

In this paper, we present an SDP relaxation for the bisection problem whose matrix variable is of order $n$. Our relaxation is equivalent to the strongest SDP relaxation for the GBP, that is the strongest Wolkowicz and Zhao (1999) relaxation. The new SDP relaxation exploits the fact that the matrix variables corresponding to the two parts in the bisection are related. Further, we consider adding the facet defining inequalities of the boolean quadric polytope to our relaxation. We also show that a large subset of the facet defining inequalities are redundant in the relaxation from Wolkowicz and Zhao (1999). The strengthened SDP bound outperforms all previously considered SDP bounds, including those tailored for highly symmetric graphs.

The paper is structured as follows. In Sect. 2 we provide an integer programming formulation of the problem, and in Sect. 3 an overview is given of the known SDP relaxations for the graph bisection problem. In Sect. 4 we present our SDP relaxation and prove that it is equivalent to the strongest SDP relaxation from Wolkowicz and Zhao (1999). We further suggest how to improve our relaxation. Finally, in Sect. 5 we present numerical results.

\section{The graph bisection problem}

In this section we formulate the minimum graph bisection problem as an integer optimization problem. Let $G=(V, E)$ be an undirected graph with vertex set $V$, where $|V|=n$ and edge set $E$. The goal is to find a partition of the vertex set into two disjoint subsets $S_{1}$ and $S_{2}$ of specified sizes $m_{1} \geq m_{2}>0, m_{1}+m_{2}=n$ such that the sum of weights of edges joining $S_{1}$ and $S_{2}$ is minimized. If $m_{1}=m_{2}$ then one refers to the associated problem as the graph equipartition problem. We consider here only the case that $m_{1}>m_{2}$. For detailed analysis of the SDP relaxations for the graph equipartition problem, see Sotirov (2012).

Let us denote by $A$ the adjacency matrix of $G$. For a given partition of the graph $G$ into two subsets, let $Z=\left(z_{i, j}\right)$ be the $n \times 2$ matrix defined by 


$$
z_{i, j}:=\left\{\begin{array}{l}
1 \text { if } i \in S_{j} \\
0 \text { otherwise }
\end{array} \quad i=1, \ldots, n, j=1,2 .\right.
$$

The $j$ th column of $Z$ is the characteristic vector of $S_{j}$. The cut of the partition, which is the sum of weights of edges joining different sets, is equal to:

$$
\frac{1}{2} \operatorname{tr}\left(A\left(J-Z Z^{\mathrm{T}}\right)\right)=\frac{1}{2} \operatorname{tr}\left(L Z Z^{\mathrm{T}}\right),
$$

where $L=\operatorname{Diag}(A e)-A$ is the Laplacian matrix of the graph, and $J$ (resp. $e$ ) the all-ones matrix (resp. vector). Therefore, the minimum GBP problem can be formulated as follows

$$
\min \left\{\frac{1}{2} \operatorname{tr}\left(L Z Z^{\mathrm{T}}\right): Z e=e, Z^{\mathrm{T}} e=m, z_{i, j} \in\{0,1\}, \forall i, j\right\},
$$

where $m=\left(m_{1}, m_{2}\right)^{\mathrm{T}}$.

\section{Overview of SDP relaxations}

In this section we provide an overview of existing SDP relaxations for the GBP. The following SDP relaxation is derived in Sotirov (2014)

$$
\begin{array}{ll}
\min & \frac{1}{2} \operatorname{tr}(L X) \\
\text { s.t. } & \operatorname{diag}(X)=e, \operatorname{tr}(J X)=m_{1}^{2}+m_{2}^{2} \\
& 2 X-J \succeq 0, X \in \mathcal{S}_{n},
\end{array}
$$

where the 'diag' operator maps an $n \times n$ matrix to the $n$-vector given by its diagonal, and $\mathcal{S}_{n}$ denotes the space of $n \times n$ symmetric matrices. Nonnegativity constraints on the matrix variable in (2) are redundant. This follows from $\operatorname{diag}(X)=e$ and $2 X-J \succeq 0$, see van Dam and Sotirov (2015) for details. The SDP relaxation (2) is equivalent to the SDP relaxation with a matrix variable of order $n$ from Karisch et al. (2000).

The following SDP relaxation for the GBP is derived in Wolkowicz and Zhao (1999):

$$
\begin{array}{ll}
\min & \frac{1}{2} \operatorname{tr}\left(L\left(Y_{11}+Y_{22}\right)\right) \\
\text { s.t. } \operatorname{tr}\left(Y_{i i}\right)=m_{i}, \operatorname{tr}\left(J Y_{i i}\right)=m_{i}^{2}, \quad i=1,2 \\
\quad \operatorname{diag}\left(Y_{12}\right)=0, \operatorname{tr} J\left(Y_{12}+Y_{12}^{\mathrm{T}}\right)=2 m_{1} m_{2} \\
\quad Y=\left(\begin{array}{ll}
Y_{11} & Y_{12} \\
Y_{12}^{\mathrm{T}} & Y_{22}
\end{array}\right) \quad y=\operatorname{diag}(Y), \quad Y-y y^{\mathrm{T}} \succeq 0, \quad Y \geq 0,
\end{array}
$$

where $Y \in \mathcal{S}_{2 n}$. From now on, we assume that matrices of order $2 n$ have the block structure as given above.

Although the nonnegativity constraints were not included in the relaxation from Wolkowicz and Zhao (1999), the authors mentioned that it would be worth adding them. The SDP relaxation (3) does not have strictly feasible solutions. From a computational point of view, this is an indication that the model may be difficult to solve directly as it is. Therefore, Wolkowicz and Zhao (1999) derive the Slater feasible version of the relaxation whose matrix variable is of order $n$. However, that model includes multiplications with projection matrices of size $(2 n+1) \times n$. The above relaxation can be further strengthened by adding the following inequalities 


$$
\begin{aligned}
0 & \leq y_{i, j} \leq y_{i, i} \\
y_{i, i}+y_{j, j} & \leq 1+y_{i, j} \\
y_{i, k}+y_{j, k} & \leq y_{k, k}+y_{i, j} \\
y_{i, i}+y_{j, j}+y_{k, k} & \leq y_{i, j}+y_{i, k}+y_{j, k}+1,
\end{aligned}
$$

where $Y=\left(y_{i, j}\right)$ and $1 \leq i, j, k \leq 2 n, i \neq j, i \neq k, j \neq k$. The inequalities (4)-(7) are facet defining inequalities of the boolean quadric polytope (BQP), see Padberg (1989).

Wolkowicz and Zhao (1999) prove that for a matrix $Y$ that is feasible for the SDP relaxation (3) the following is satisfied:

$$
Y_{11}+Y_{12}=y_{1} e^{\mathrm{T}}, \quad Y_{12}^{\mathrm{T}}+Y_{22}=y_{2} e^{\mathrm{T}}, \quad y_{1}+y_{2}=e, \quad Y_{i i} e=m_{i} y_{i}(i=1,2),
$$

where $y_{i}=\operatorname{diag}\left(Y_{i i}\right)$. From here it follows that for given $Y_{11}$ and $y_{1}$ the above equations uniquely determine $Y_{12}, Y_{22}$ and $y_{2}$. We will exploit this to derive the simplified SDP relaxation in the following section.

Extensive numerical results in Sotirov (2014) show that (3) provides the strongest SDP relaxation for the GBP. To the best of our knowledge, we are not aware of numerical test that involve the SDP relaxation (3) and the inequalities (4)-(7).

Finally, we prove that the optimal value of the SDP relaxation (3) is at least that of the relaxation (2).

Proposition 1 Let $m_{1}>m_{2}$ and $m_{1}+m_{2}=n$. Then the SDP relaxation (3) dominates the $S D P$ relaxation (2).

Proof Let $Y_{i j}$ and $y_{i}(i, j=1,2)$ be feasible for (3), and set $X=Y_{11}+Y_{22}$. Now, $\operatorname{tr}(J X)=$ $m_{1}^{2}+m_{2}^{2}$ and $\operatorname{diag}(X)=e$ follow from feasibility of $Y_{i i}(i=1,2)$ and (8). The SDP constraint follows from summing $\left(\begin{array}{cc}Y_{i i} & y_{i} \\ y_{i}^{\mathrm{T}} & 1\end{array}\right) \succeq 0, i=1,2$.

The similar result is proven in De Klerk et al. (2012). In particular, it was proven that the QAP-based SDP relaxation for the GBP dominates the SDP relaxation from Karisch et al. (2000). However, the QAP-based SDP relaxation for the GBP is equivalent to (3), and the relaxation from Karisch et al. (2000) to (2), see Sotirov (2014).

\section{A simplified SDP relaxation}

In this section we derive an SDP relaxation for the GBP with a matrix variable of order $n$, and prove that it is equivalent to the best known SDP relaxation for general graphs that is derived in Wolkowicz and Zhao (1999). To derive the relaxation we exploit the fact that the variables associated to the two sets in the bisection are related. Namely, variables coming from the assignment to the second set are redundant in the assignment constraints. It is surprising that this observation was not earlier exploited in the context of the GBP. However, a similar idea was used in Rendl and Sotirov (2016) to derive an SDP relaxation for the vertex separator problem.

Our observation lead us to the following SDP relaxation:

$$
\begin{array}{ll}
\min & \frac{1}{2} \operatorname{tr}\left(L\left(2 X+J-x e^{\mathrm{T}}-e x^{\mathrm{T}}\right)\right) \\
\text { s.t. } & x^{\mathrm{T}} e=m_{1}, \quad \operatorname{tr}(J X)=m_{1}^{2}, \quad X e=m_{1} x \\
& X \geq 0, \quad x e^{\mathrm{T}}-X \geq 0, \quad J+X-x e^{\mathrm{T}}-e x^{\mathrm{T}} \geq 0 \\
& X \geq 0, \quad \operatorname{diag}(X)=x, \quad X \in \mathcal{S}_{n} .
\end{array}
$$


All equality constraints in (9) are related to the variables associated to the set $S_{1}$. The constraints $X \geq 0$ ensure that the matrix variable corresponding to $S_{1}$ is nonnegative, while constraints $x e^{\mathrm{T}}-X \geq 0, J+X-x e^{\mathrm{T}}-e x^{\mathrm{T}} \geq 0$ do the same for the slack matrix variables.

One may wish to replace the semidefinite constraint $X \succeq 0$ from (9) with the in general stronger constraint $X-x x^{\mathrm{T}} \succeq 0$. However, from the following result it follows that in our case those two semidefinite constraints are equivalent.

Proposition 2 (Gijswijt 2005, Proposition 7) Let X be a symmetric matrix of order $n$ such that $c \cdot \operatorname{diag}(X)=X$ for some $c \in \mathbf{R}$, and

$$
\bar{X}=\left(\begin{array}{cc}
1 & \operatorname{diag}(X)^{\mathrm{T}} \\
\operatorname{diag}(X) & X
\end{array}\right) .
$$

Then the following are equivalent:

(i) $\bar{X}$ is positive semidefinite,

(ii) $X$ is positive semidefinite and $\operatorname{tr}(J X) \geq(\operatorname{tr} X)^{2}$.

The equivalence of the two SDP constraints follows from the fact that for a feasible $X$ for (9) one has $\operatorname{tr}(J X)=(\operatorname{tr} X)^{2}=m_{1}^{2}$ and $X e=m_{1} \operatorname{diag}(X)$. We prove now our main result.

Theorem 3 Let $m_{1}+m_{2}=n, m_{1}>m_{2}$. The SDP relaxations (3) and (9) are equivalent.

Proof Let $X$ be feasible for (9) and $x=\operatorname{diag}(X)$. We construct a feasible $Y, y=\operatorname{diag}(Y)$ for (3) in the following way. Define $y_{1}:=x, y_{2}:=e-x, y^{\mathrm{T}}:=\left(y_{1}^{\mathrm{T}}, y_{2}^{\mathrm{T}}\right)$, matrices

$$
Y_{11}:=X, \quad Y_{22}:=J+X-x e^{\mathrm{T}}-e x^{\mathrm{T}}, \quad Y_{12}:=x e^{\mathrm{T}}-X,
$$

and collect all blocks into the matrix

$$
Y=\left(\begin{array}{ll}
Y_{11} & Y_{12} \\
Y_{12}^{\mathrm{T}} & Y_{22}
\end{array}\right)=\left(\begin{array}{cc}
X & x e^{\mathrm{T}}-X \\
e x^{\mathrm{T}}-X & J+X-x e^{\mathrm{T}}-e x^{\mathrm{T}}
\end{array}\right) .
$$

Now, we first prove that

$$
\left(\begin{array}{cc}
X & x e^{\mathrm{T}}-X \\
e x^{\mathrm{T}}-X & J+X-x e^{\mathrm{T}}-e x^{\mathrm{T}}
\end{array}\right)-\left(\begin{array}{cc}
x x^{\mathrm{T}} & x(e-x)^{\mathrm{T}} \\
(e-x) x^{\mathrm{T}} & (e-x)(e-x)^{\mathrm{T}}
\end{array}\right) \succeq 0 .
$$

To show this, we rewrite the left hand side of the matrix inequality above as it follows

$$
\left(\begin{array}{ll}
X-x x^{\mathrm{T}} & x x^{\mathrm{T}}-X \\
x x^{\mathrm{T}}-X & X-x x^{\mathrm{T}}
\end{array}\right)
$$

Now, for arbitrary vectors $z_{1}, z_{2} \in \mathbf{R}^{n}$ we have

$$
\left(z_{1}^{\mathrm{T}}, z_{2}^{\mathrm{T}}\right)\left(\begin{array}{ll}
X-x x^{\mathrm{T}} & x x^{\mathrm{T}}-X \\
x x^{\mathrm{T}}-X & X-x x^{\mathrm{T}}
\end{array}\right)\left(\begin{array}{l}
z_{1} \\
z_{2}
\end{array}\right)=\left(z_{1}-z_{2}\right)^{\mathrm{T}}\left(X-x x^{\mathrm{T}}\right)\left(z_{1}-z_{2}\right) \geq 0,
$$

from where it follows the claim. Let us now verify $\operatorname{tr}\left(J Y_{22}\right)=m_{2}^{2}$. Namely,

$$
\operatorname{tr}\left(J Y_{22}\right)=\operatorname{tr}\left(J\left(J+X-x e^{\mathrm{T}}-e x^{\mathrm{T}}\right)\right)=n^{2}+m_{1}^{2}-2 n m_{1}=m_{2}^{2} .
$$

Similarly, the remaining constraints from (3) can be verified.

Conversely, let $Y$ be feasible for (3). We set $X=Y_{11}$ and $x=\operatorname{diag}\left(Y_{11}\right)$. Since every feasible matrix $Y \in \mathcal{S}_{2 n}$ for (3) satisfies also (8), feasibility of $X$ follows by direct verification. Finally, it is not difficult to check that the objectives coincide for any pair of feasible solutions $(Y, X)$. 
Note that the result from the previous theorem is also valid when $m_{1}=m_{2}$. However, it was proven in Sotirov (2012) that all known vector and matrix lifting based SDP relaxations for the $k$-equipartition problem $(k \geq 2)$ are equivalent.

It is not difficult to verify that the SDP relaxation (9) has a strictly feasible point. Indeed, the following matrix is feasible for (9) and is positive definite:

$$
\hat{X}=\frac{m_{1}}{n} I+\frac{m_{1}\left(m_{1}-1\right)}{n(n-1)}(J-I),
$$

where $I$ is the identity matrix. Note that $\hat{X}$ has two distinct eigenvalues, i.e., $\frac{m_{1}\left(n-m_{1}\right)}{n(n-1)}$ with multiplicity $n-1$, and $\frac{m_{1}^{2}}{n}$ with multiplicity one.

The following result is a direct consequence of Theorem 3.

Corollary 4 The SDP relaxation (3) without nonnegativity constraints is equivalent to the $S D P$ relaxation (9) without nonnegativity constraints, i.e.,

$$
\begin{aligned}
& \min \frac{1}{2} \operatorname{tr}\left(L\left(2 X+J-x e^{\mathrm{T}}-e x^{\mathrm{T}}\right)\right) \\
& \text { s.t. } x^{\mathrm{T}} e=m_{1}, \quad \operatorname{tr}(J X)=m_{1}^{2}, \quad X e=m_{1} x \\
& \quad X \succeq 0, \quad \operatorname{diag}(X)=x, \quad X \in \mathcal{S}_{n} .
\end{aligned}
$$

In order to improve the SDP relaxation (9) we can add the facet defining inequalities of the boolean quadric polytope, see Padberg (1989). We first note that the inequality constraints $X \geq 0, x e^{\mathrm{T}}-X \geq 0$, and $J+X-x e^{\mathrm{T}}-e x^{\mathrm{T}} \geq 0$ from the SDP relaxation (9) are exactly the following BQP constraints

$$
0 \leq x_{i, j} \leq x_{i, i}, \quad x_{i, i}+x_{j, j} \leq 1+x_{i, j}, \quad 1 \leq i, j \leq n, i \neq j .
$$

Note also that the SDP relaxation from Corollary 4 differs from the SDP relaxation (9) exactly for those constraints. Thus, in order to strengthen the SDP relaxation (9) one can add the following BQP constraints:

$$
x_{i, k}+x_{j, k} \leq x_{k, k}+x_{i, j}, \quad x_{i, i}+x_{j, j}+x_{k, k} \leq x_{i, j}+x_{i, k}+x_{j, k}+1,
$$

for $1 \leq i, j, k \leq n, i \neq j, i \neq k, j \neq k$.

Let us now show that the bound obtained by solving the SDP relaxation (3) with additional BQP constraint (4)-(7) is equal to the bound obtained by solving (9) with additional constraints (10). We first prove that (4)-(5) are redundant for feasible matrices from (3).

Lemma 5 Let $Y=\left(y_{i, j}\right) \in \mathcal{S}_{2 n}$ be feasible for (3). Then, the following BQP inequalities are satisfied:

$$
0 \leq y_{i, j} \leq y_{i, i}, \quad y_{i, i}+y_{j, j} \leq 1+y_{i, j},
$$

for $1 \leq i, j \leq 2 n, i \neq j$.

Proof The constraints $0 \leq y_{i, j} \leq y_{i, i}(1 \leq i, j \leq 2 n)$ follow trivially from (8) and $Y \geq 0$. To show that the following inequalities

$$
y_{i, i}+y_{j, j} \leq 1+y_{i, j}, \quad 1 \leq i, j \leq n, i \neq j,
$$

are satisfied, it is instructive to look at (8). From (8) we have that $Y_{22}=J+Y_{11}-y_{1} e^{\mathrm{T}}-e y_{1}^{\mathrm{T}}$. Now, from $Y_{22} \geq 0$ it follows that $J+Y_{11} \geq y_{1} e^{\mathrm{T}}+e y_{1}^{\mathrm{T}}$, from where it is clear that the above constraints are satisfied. To verify the inequalities

$$
y_{i, i}+y_{j, j} \leq 1+y_{i, j}, \quad 1 \leq i \leq n, \quad n+1 \leq j \leq 2 n,
$$


we rewrite $y_{j, j}(n+1 \leq j \leq 2 n)$ in the terms of the elements from the first block by using $Y_{22}=J+Y_{11}-y_{1} e^{\mathrm{T}}-e y_{1}^{\mathrm{T}}$. Similarly, we rewrite $y_{i, j}(1 \leq i \leq n, n+1 \leq j \leq 2 n)$ using $Y_{12}=y_{1} e^{\mathrm{T}}-Y_{11}$. Now, the above inequalities reduce to the redundant constraints $y_{k, k} \geq y_{k, i}(1 \leq i, k \leq n)$.

Similarly, one can show that $y_{i, i}+y_{j, j} \leq 1+y_{i, j}$ for $n+1 \leq i, j \leq 2 n$, and $n+1 \leq i \leq 2 n$, $1 \leq j \leq n$.

In the previous lemma we exploit the fact that a feasible matrix for (3) satisfies (8). By using the same equations, we can show that for a feasible $Y$ from (3) many of the constraints (6)-(7) are equivalent. In particular, let $Y=\left(y_{i, j}\right) \in \mathcal{S}_{2 n}$ be feasible for (3). Then the QBP constraints (6)-(7) for the elements in the blocks $Y_{12}, Y_{12}^{\mathrm{T}}$ and $Y_{22}$ can be reformulated into the QBP constraints (6)-(7) for the elements in the block $Y_{11}$. For example, to reformulate the inequalities

$$
y_{i, k}+y_{j, k} \leq y_{k, k}+y_{i, j}, \quad 1 \leq i \leq n, n+1 \leq j, k \leq 2 n, j \neq k,
$$

we exploit $Y_{22}=J+Y_{11}-y_{1} e^{\mathrm{T}}-e y_{1}^{\mathrm{T}}$ to rewrite $y_{j, k}$ and $y_{k, k}$. We also use $Y_{12}=y_{1} e^{\mathrm{T}}-Y_{11}$ to rewrite $y_{i, k}$ and $y_{i, j}$, which leads to the inequalities

$$
y_{i, j}+y_{k, j} \leq y_{j, j}+y_{i, k}, \quad 1 \leq i, j, k \leq n .
$$

Similarly, to reformulate the inequalities

$$
y_{i, i}+y_{j, j}+y_{k, k} \leq y_{i, j}+y_{i, k}+y_{j, k}+1, n+1 \leq i, j, k \leq 2 n, j \neq k \neq i,
$$

we exploit $Y_{22}=J+Y_{11}-y_{1} e^{\mathrm{T}}-e y_{1}^{\mathrm{T}}$ to rewrite all $y$-variables. This results with the constraints:

$$
y_{i, i}+y_{j, j}+y_{k, k} \leq y_{i, j}+y_{i, k}+y_{j, k}+1, \quad 1 \leq i, j, k \leq n, j \neq k \neq i .
$$

Continuing in a similar way, we get that the constraints (6)-(7) can be reduced to the constraints (11)-(12), i.e., to the same inequalities for the smaller index set. We summarize the previous results in the following theorem.

Theorem 6 The SDP relaxation (9) with additional constraints (10) is equivalent to the SDP relaxation (3) with additional BQP constraints (4)-(7).

Thus, this paper presents reformulated and simplified the strongest SDP relaxation for the the bisection problem, and suggest its strengthening. In the following section we test our simplified and strengthened SDP relaxation on several graphs from the literature.

\section{Numerical results}

In this section we present numerical results that verify the quality of the SDP relaxation (9), as well as the relaxation obtained after adding the BQP constraints (10) to (9). All relaxations were solved with MOSEK Aps (2015) using the Yalmip interface (Löfberg 2004) on an Intel Xeon, E5-1620, 3.70 GHz with 32 GB memory.

The instances we use belong to the various classes of graphs from the literature. In particular, in Tables 1 and 2 we consider the following graphs.

- compiler design instances were introduced in Johnson et al. (1993). We denote them by cd.xx.yy. 
Table 1 Computational results for the bisection problem

\begin{tabular}{|c|c|c|c|c|c|c|}
\hline Instance & $|V|$ & $m^{\mathrm{T}}$ & (2) & (9) & $(9)+(10)$ & u.b. \\
\hline cd.30.47 & 30 & $(20,10)$ & 110 & 114 & 114 & 114 \\
\hline cd.30.56 & 30 & $(20,10)$ & 156 & 169 & 169 & 169 \\
\hline cd.45.98 & 45 & $(25,20)$ & 576 & 631 & 631 & 631 \\
\hline cd.47.99 & 47 & $(25,22)$ & 471 & 514 & 537 & 537 \\
\hline cd.47.101 & 47 & $(25,22)$ & 326 & 361 & 382 & 382 \\
\hline cd.61.187 & 61 & $(40,21)$ & 774 & 798 & 798 & 798 \\
\hline kkt_lowt 01 & 82 & $(42,40)$ & 5 & 5 & 13 & 13 \\
\hline kkt_putt01 & 115 & $(59,56)$ & 20 & 22 & 28 & 29 \\
\hline mesh.35.54 & 35 & $(22,13)$ & 2 & 3 & 4 & 4 \\
\hline mesh.69.212 & 69 & $(40,29)$ & 2 & 2 & 4 & 4 \\
\hline mesh.70.120 & 70 & $(50,20)$ & 2 & 4 & 6 & 6 \\
\hline mesh.74.129 & 74 & $(70,4)$ & 1 & 4 & 4 & 4 \\
\hline mesh.137.231 & 137 & $(100,37)$ & 1 & 3 & 6 & 6 \\
\hline mesh.148.265 & 148 & $(120,28)$ & 1 & 5 & 6 & 6 \\
\hline vlsi.34.71 & 34 & $(22,12)$ & 4 & 6 & 6 & 6 \\
\hline vlsi.37.92 & 37 & $(30,7)$ & 3 & 6 & 6 & 6 \\
\hline vlsi.38.105 & 38 & $(20,18)$ & 84 & 86 & 110 & 110 \\
\hline vlsi.42.132 & 42 & $(20,22)$ & 97 & 99 & 120 & 120 \\
\hline vlsi.48.81 & 48 & $(40,8)$ & 4 & 12 & 12 & 18 \\
\hline vlsi.166.504 & 166 & $(100,66)$ & 12 & 23 & 24 & 24 \\
\hline vlsi.170.424 & 170 & $(100,70)$ & 35 & 37 & 37 & 48 \\
\hline
\end{tabular}

Table 2 Computational times in seconds for the bisection problem

\begin{tabular}{|c|c|c|c|c|c|c|}
\hline Instance & $|V|$ & $m^{\mathrm{T}}$ & (2) & (9) & (3) & $(9)+(10)$ \\
\hline cd.47.99 & 47 & $(25,22)$ & 1 & 2 & 2 & 7 \\
\hline cd.47.101 & 47 & $(25,22)$ & 1 & 2 & 3 & 6 \\
\hline cd.61.187 & 61 & $(40,21)$ & 3 & 4 & 4 & n.a. \\
\hline kkt_lowt01 & 82 & $(42,40)$ & 10 & 21 & 21 & 442 \\
\hline kkt_putt01 & 115 & $(59,56)$ & 66 & 106 & 114 & 1289 \\
\hline mesh.35.54 & 35 & $(22,13)$ & 1 & 1 & 1 & 3 \\
\hline mesh. 69.212 & 69 & $(40,29)$ & 6 & 9 & 11 & 83 \\
\hline mesh.70.120 & 70 & $(50,20)$ & 4 & 12 & 9 & 55 \\
\hline mesh.74.129 & 74 & $(70,4)$ & 7 & 12 & 14 & n.a. \\
\hline mesh.137.231 & 137 & $(100,37)$ & 167 & 296 & 345 & 6574 \\
\hline mesh.148.265 & 148 & $(120,28)$ & 241 & 550 & 566 & 990 \\
\hline vlsi.38.105 & 38 & $(20,18)$ & 1 & 1 & 1 & 15 \\
\hline vlsi.42.132 & 42 & $(20,22)$ & 1 & 1 & 1 & 20 \\
\hline vlsi.48.81 & 48 & $(40,8)$ & 1 & 2 & 2 & 11 \\
\hline vlsi.166.504 & 166 & $(100,66)$ & 371 & 892 & 1081 & 2079 \\
\hline vlsi.170.424 & 170 & $(100,70)$ & 486 & 943 & 1252 & 7789 \\
\hline
\end{tabular}


- kkt instances originate from nested dissection approaches for solving sparse symmetric linear systems, see Helmberg (2004). We denote them by kkt_name.

- mesh instances come from an application of the finite element methods, see Souza et al. (1994). We denote them with the initials mesh.xx.yy.

- VLSI design instances are derived from data in the layout of electronic circuits. For details see Ferreira et al. (1998). We denote them with the initials vlsi . xx . Yy.

In the above instances $x x$ denotes the number of vertices, and yy the number of edges in the graph. Table 1 reads as follows. In the first three columns, we list the graphs, number of vertices in the graph, and corresponding $m$, respectively. In the fourth to six column we present the SDP bounds (2), (9), and the SDP bound (9) with additional BQP constraints (10), respectively. Bounds in the column six are obtained by adding the most violated inequalities of type (10) to the SDP relaxation (9). The cutting plane scheme adds at most $2 n$ violated valid constraints in each iteration and performs at most 20 iterations. In the last column of Table 1 we list upper bounds obtained by a tabu search heuristics, see also Rolland et al. (1996).

All lower bounds in Table 1 are rounded up to the closest integer. Note that for only three out of twenty-one instances we can not prove optimality.

In Table 2 we list the computational times required for solving the SDP relaxations and instances from Table 1. In the same table we include the computational times for solving the Slater feasible version of the SDP relaxation (3), see Wolkowicz and Zhao (1999). We do not compute the SDP bound (9)+(10), if (9) provides an optimal solution. In such cases, we write 'n.a.'. Since the computational times for computing each of the lower bounds for cd.30.47, cd.30.56, cd.45.98 are below $1 \mathrm{~s}$, we omit these results from the table.

Table 2 shows that there is only a marginal time difference for solving (2), (9), and (3) for graphs up to 61 vertices. The results show that for graphs with more than 61 vertices, the SDP relaxation (2) requires noticeable less computational effort than the other relaxations. Computational times in Table 2 verify that there is an advantage in solving the relaxation (9) for larger graphs $(n>115)$ than solving (3).

Table 2 indicates that sometimes the running time for solving (9)+(10) is a few times longer than the running time for solving (9). For example, we compute the bound (9) for vlsi.166.504 $(n=166)$ in $892 \mathrm{~s}$, and (9)+(10) in 2079 s. See also results for cd.47.101, mesh.148.265. However, the difference between the two running times can be significant. In particular, we compute the SDP bound (9) for kkt_putt01 $(n=115)$ in $106 \mathrm{~s}$, and $(9)+(10)$ in $21 \mathrm{~min}$. [To approximately solve the SDP relaxation (3)+(4)-(7) using the cutting plane schema for kkt_putt01 it takes about $47 \mathrm{~min}]$. In general, the computational time for solving (9) $+(10)$ depends on the number of the violated BQP constraints (10) after each iteration of the cutting plane scheme.

Van Dam and Sotirov (2015) strengthened the SDP relaxations (2) and (3) by adding two constraints that correspond to assigning two vertices of the graph to different parts of the partition. In particular, they show that such strengthening performs well on highly symmetric graphs when other relaxations provide weak or trivial bounds. In van Dam and Sotirov (2015), it was also shown how to aggregate the triangle and independent set constraints for highly symmetric graphs in order to add them to the SDP relaxation (2). Our numerical results show that the SDP relaxation (9) with additional inequalities (10) provides bounds that are competitive to those from van Dam and Sotirov (2015).

In particular, in Table 3 we list bounds for highly symmetric graphs considered in van Dam and Sotirov (2015). Pappus, Desargues, and Biggs-Smith graphs are distanceregular graphs, $J(7,2)$ is the Johnson graph. The first three columns in Table 3 read similar 
Table 3 Bounds for the bisection on highly symmetric graphs

\begin{tabular}{lclllrr}
\hline$G$ & $|V|$ & $m^{\mathrm{T}}$ & $(9)$ & $\begin{array}{l}\text { b.b. (van Dam and } \\
\text { Sotirov 2015) }\end{array}$ & $\begin{array}{l}(9)+(10) \\
\text { u.b. }\end{array}$ \\
\hline Pappus & 18 & $(10,8)$ & 6 & 7 & 7 & 8 \\
Desargues & 20 & $(15,5)$ & 5 & 6 & 6 & 7 \\
$J(7,2)$ & 21 & $(11,10)$ & 37 & 40 & 40 & 40 \\
Biggs-Smith & 102 & $(70,32)$ & 10 & 15 & 15 & 18 \\
\hline
\end{tabular}

as the first three columns in Table 1. In the fourth (resp. sixth) column we list values of the SDP bound (9) (resp. bound (9) with additional inequalities (10)) for different graphs. The fifth column of Table 3 lists the best obtained bounds from van Dam and Sotirov (2015); that is for the Pappus graph the relaxation (2) with all triangle inequalities, for Desargues the relaxation (3) with constraints that fix two vertices of the graph, for $J(7,2)$ the relaxation (2) with independent set inequalities, and for Biggs-Smith the relaxation (2) with all triangle inequalities.

Table 3 shows that our new relaxation (9) +(10) provides lower bounds that are competitive with other bounding approaches known for highly symmetric graphs. Although most of the bounds presented in van Dam and Sotirov (2015) can be solved within a few seconds, it is not clear a priori which of the bounding approaches should be implemented for a given graph. However, the SDP relaxation $(9)+(10)$ provides lower bounds that equal the best bounds among all approaches studied in van Dam and Sotirov (2015). To compute the SDP bound (9) for Biggs-Smith it takes $22 \mathrm{~s}$, and to compute the bound (9) + (10) for $J(7,2)$ (resp. Biggs-Smith) it takes $4 \mathrm{~s}$ (resp. $773 \mathrm{~s}$ ). All other bounds in Table 3 are obtained within a second. To compute the SDP bound (9) for highly symmetric graphs we didn't exploit symmetry reduction as described in van Dam and Sotirov (2015) although this can be done in a similar way. By doing as described in van Dam and Sotirov (2015), one can compute SDP bounds (9) from Table 3 very fast. The interested reader is invited to verify this.

\section{Conclusion}

In this paper we present an SDP relaxation for the graph bisection problem with a matrix variable of order $n$, where $n$ is the order of the graph. To derive our relaxation we exploit the fact that variables corresponding to one set in the bisection uniquely determine variables of the other set. We prove that our relaxation is equivalent to the strongest known SDP relaxation for general graphs that is obtained by using vector lifting. This result is in the line of the similar results for some other optimization problems. Namely, for the graph equipartition problem there exists an SDP relaxation with a matrix variable of order equal to the order of the graph, which is equivalent to the strongest vector lifting-based SDP relaxation, see Sotirov (2012).

To strengthen our SDP relaxation we add facet defining inequalities of the boolean quadric polytope, which enables us to compute strongest SDP bounds for the GBP and for graphs with $n \leq 200$ vertices in reasonable time.

Since our relaxation has strictly feasible solutions it can be directly solved as it is, which makes it attractive for a branch and bound framework. However, this will be part of our future research. 
Acknowledgements The author would like to thank two anonymous referees for suggestions that led to an improvement of this paper.

Open Access This article is distributed under the terms of the Creative Commons Attribution 4.0 International License (http://creativecommons.org/licenses/by/4.0/), which permits unrestricted use, distribution, and reproduction in any medium, provided you give appropriate credit to the original author(s) and the source, provide a link to the Creative Commons license, and indicate if changes were made.

\section{References}

Biswas, R., Hendrickson, B., \& Karypis, G. (2000). Graph partitioning and parallel computing. Parallel Computing, 26(12), 1515-1517.

Dai, W., \& Kuh, E. (1987). Simultaneous floor planning and global routing for hierarchical building-block layout. IEEE Transaction on Computer-Aided Design Integrated Circuits \& Systems CAD, 6(5), 828-837.

De Klerk, E., Pasechnik, D. V., Sotirov, R., \& Dobre, C. (2012). On semidefinite programming relaxations of maximum $k$-section. Mathematical Programming Series B, 136(2), 253-278.

De Klerk, E., de Oliveira Filho, F. M., \& Pasechnik, D. V. (2012). Relaxations of combinatorial problems via association schemes. In M. Anjos \& J. Lasserre (Eds.), Handbook of semidefinite, cone and polynomial optimization: Theory, algorithms, software and applications (pp. 171-200). New York: Springer.

de Souza, C. C., Keunings, R., Wolsey, L. A., \& Zone, O. (1994). A new approach to minimizing the frontwidth in finite element calculations. Computer Methods in Applied Mechanics and Engineering, 111, 323-334.

Feige, U., \& Langberg, M. (2001). Approximation algorithms for maximization problems arising in graph partitioning. Journal of Algorithms, 41, 174-211.

Ferreira, C. E., Martin, A., de Souza, C. C., Weismantel, R., \& Wolsey, L. A. (1998). The node capacitated graph partitioning problem: A computational study. Mathematical Programming, 81, 229-256.

Fiduccia, C. M., \& Mattheyses, R. M. (1982). A linear-time heuristic for improving network partitions. In Proceedings of the 19th Design Automation Conference (pp. 175-181).

Garey, M. R., Johnson, D. S., \& Stockmeyer, L. (1976). Some simplified NP-complete graph problems. Theoretical Computer Science, 1(3), 237-267.

Gijswijt, D. (2005). Matrix algebras and semidefinite programming techniques for codes. PhD thesis, University of Amsterdam, The Netherlands.

Han, Q., Ye, Y., \& Zhang, J. (2002). An improved rounding method and semidefinite relaxation for graph partitioning. Mathematical Programming, 92, 509-535.

Helmberg, C. (2004). A cutting plane algorithm for large scale semidefinite relaxations. In M. Grötschel (Ed.), Padberg Festschrift the sharpest cut (pp. 233-256). MPS-SIAM.

Hendrickson, B., \& Kolda, T. G. (2000). Partitioning rectangular and structurally nonsymmetric sparse matrices for parallel processing. SIAM Journal of Scientific Computing, 21(6), 2048-2072.

Johnson, E., Mehrotra, A., \& Nemhauser, G. (1993). Min-cut clustering. Mathematical Programming, 62, $133-152$.

Karisch, S. E., Rendl, F., \& Clausen, J. (2000). Solving graph bisection problems with semidefinite programming. INFORMS Journal on Computing, 12, 177-191.

Lengauer, T. (1990). Combinatorial algorithms for integrated circuit layout. Chicester: Wiley.

Li, M., Andersen, D. G., \& Smola, A. J. (2015). Graph partitioning via parallel submodular approximation to accelerate distributed machine learning. arXiv:1505.04636v1.

Löfberg, J. (2004). YALMIP: A toolbox for modeling and optimization in MATLAB. In Proceedings of the CACSD Conference, Taipei, Taiwan (pp. 284-289). http://users.isy.liu.se/johanl/yalmip/.

MOSEK Aps. (2015). The MOSEK optimization toolbox for MATLAB manual. Version 7.1 (Revision 28) http://docs.mosek.com/7.1/toolbox/index.html.

Padberg, M. W. (1989). The boolean quadric polytope: Some characteristics, facets and relatives. Mathematical Programming, 45, 139-172.

Pirim, H., Ekçioğlu, B., Perkins, A., \& Yüceer, Ç. (2012). Clustering of high throughput gene expression data. Computers \& Operations Research, 39(12), 3046-3061.

Rendl, F., \& Sotirov, R. (2016). The min-cut and vertex separator problem, Preprint. http://www.optimizationonline.org/DB_FILE/2016/01/5278.pdf.

Rolland, E., Pirkul, H., \& Glover, F. (1996). Tabu search for graph partitioning. Annals of Operations Research, 63(2), 209-232.

Sanchis, L. (1989). Multiple-way network partitioning. IEEE Transaction on Computers, 38, 62-81. 
Simon, H. D. (1991). Partitioning of unstructured problems for parallel processing. Computing Systems in Engineering, 2, 35-148.

Sotirov, R. (2012). SDP relaxations for some combinatorial optimization problems. In M. F. Anjos \& J. B. Lasserre (Eds.), Handbook of semidefinite, conic and polynomial optimization: Theory, algorithms, software and applications (pp. 795-820). New York: Springer.

Sotirov, R. (2014). An efficient semidefinite programming relaxation for the graph partition problem. INFORMS Journal of Computers, 26(1), 16-30.

van Dam, E. R., \& Sotirov, R. (2015). Semidefinite programming and eigenvalue bounds for the graph partition problem. Mathematical Programming Series B, 151(2), 379-404.

Wolkowicz, H., \& Zhao, Q. (1999). Semidefinite programming relaxations for the graph partitioning problem. Discrete Applied Mathematics, 96(97), 461-479. 